\title{
SINDROME DE PARSONAGE-TURNER O NEURITIS BRAQUIAL: A PROPOSITO DE DOS CASOS CLINICOS
}

\section{Drs. Luis Muse R, Oscar Contreras 0.}

Servicio de Radiología, Hospital Clínico de la Universidad Católica de Chile.

\begin{abstract}
Two cases of Parsonage-Turner syndrome are presented. The clinical presentation and MRI characteristics are discussed.
\end{abstract}

Key words: Neuritis, MRI, Shoulder.

Resumen: Se presentan dos casos clínicos de neuritis braquial (síndrome de Parsonage-Turner). Se discuten los aspectos clínicos y los hallazgos en resonancia magnética destacando el valor de ésta en su diagnóstico.

Palabras claves: Hombro, Neuritis, Resonancia magnética.

\section{Introducción}

La neuritis braquial, también conocida como síndrome de Parsonage-Turner es una rara alteración neuromuscular del hombro, que se caracteriza por un dolor intenso de comienzo brusco, que posteriormente se acompaña de debilidad muscular. Su etiología es aún desconocida. El diagnóstico principalmente se basaba en la historia clínica y en los resultados obtenidos en la electromiografía.

Presentamos dos casos de neuritis braquial, haciendo énfasis en la utilidad de la resonancia magnética $(\mathrm{RM})$ en el diagnóstico.

\section{Casos clínicos}

Caso 1. Hombre de 50 años que presenta un dolor agudo del hombro derecho horas después de haber sido operado de una gastroplastía. El dolor es permanente y progresivo, no se asocia a déficit neurológico. Se le realiza una radiografía de hombro que fue normal y posteriormente una RM (Figura 1).

Muse L. Contreras O. Síndrome de Parsonage-Turner o neuritis braquial: A propósito de dos casos clínicos. Rev Chil Radiol 2003; 9: 137-139.

Correspondencia: Drs. Luis Muse R, Oscar Contreras $O$. Servicio de Radiología, Hospital Clínico de la Universidad Católica de Chile. Marcoleta 371. Santiago, Chile. ocontrer@met.puc.cl
Caso 2. Hombre de 30 años que 5 días después de un leve trauma en el hombro, izquierdo comienza con un dolor agudo, que no remite con manejo de analgésicos, por lo tanto se indica el estudio con RM (Figura 2).

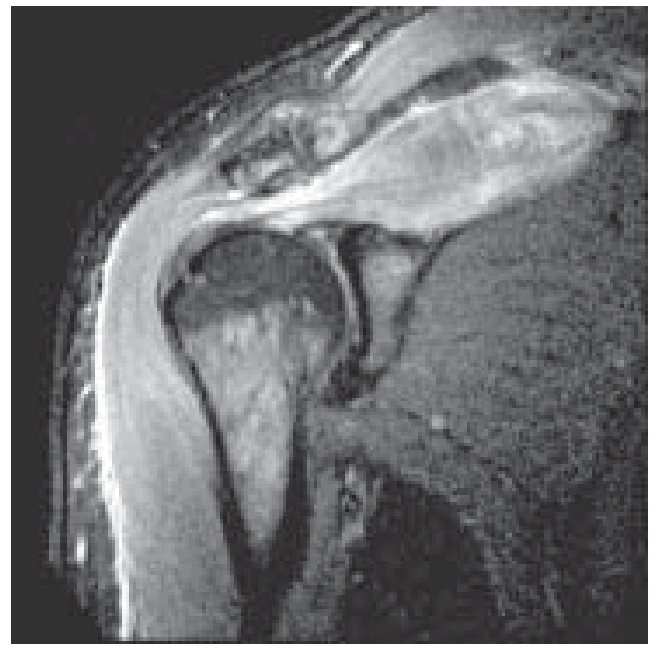

Figura 1. RM secuencia STIR corte coronal de hombro derecho. Nótese el aumento en la intensidad de señal en el músculo supraespinoso reflejando edema en relación a él.

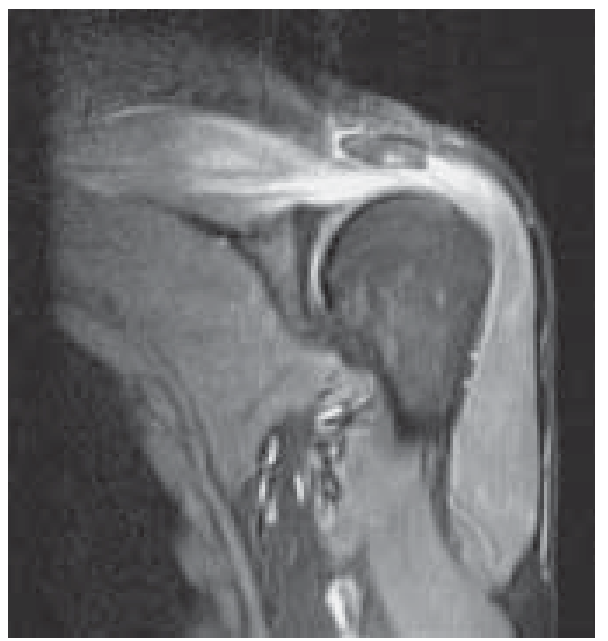

Figura 2. RM secuencia STIR corte coronal de hombro izquierdo, demostrando aumento de señal a nivel de músculos supraespinoso y deltoides. 


\section{Discusión}

El síndrome de Parsonage-Turner es una alteración rara de etiología desconocida, que se caracteriza por un dolor agudo e intenso del hombro y de la extremidad superior, seguida de paresia y atrofia muscular.

La primera descripción fue realizada en 1987 por Feinberg, quien relata un caso de neuritis braquial unilateral completa del plexo braquial asociado a influenza. El más importante reporte fue el realizado por Spillane ${ }^{(6)}$, quien reportó una neuritis localizada del hombro en 46 soldados en el medio oeste.

En 1948 Parsonage y Turner ${ }^{(7)}$ publicaron los hallazgos en 136 soldados durante la Segunda Guerra Mundial. Otro estudio de estos mismos autores realizado en 1957, revelo similares hallazgos en 82 pacientes en Londres, (Inglaterra).

Una incidencia de 1.64 casos por 100.000 personas, fue reportada en un estudio realizado por Beghi y col $^{(8)}$, quien utilizó la base de datos de la Clínica Mayo. La edad de los pacientes con neuritis braquial, tuvo un rango que fluctuó entre 3 meses y 75 años, la máxima incidencia estuvo entre la tercera y séptima década de vida. Los hombres son más afectados que las mujeres, con un rango de 2:1 a 11,5:1. No se ha visto predominancia de los síntomas de un lado sobre el otro, así como tampoco existiría una mayor frecuencia en la en la extremidad dominante. Se ha visto que en un tercio de los pacientes, los síntomas se presentan bilateralmente y en forma simétrica.

Numerosos autores han especulado acerca de la etiología de la neuritis braquial. Además se han descrito varios eventos o factores que precipitarían esta condición, por ejemplo el trauma, infección, ejercicios pesados, cirugías, inmunización y mecanismos autoinmunes. En relación al trauma se demostró en un reporte realizado por Parsonage y Turner $^{(7)}$ que 5 de 136 pacientes presentaron como antecedente un leve trauma en el hombro o en la región cervical, aproximadamente una semana antes del comienzo de los síntomas. Esta situación se observó en uno de nuestros pacientes.

En relación a infecciones se ha visto que precede al comienzo de los síntomas en aproximadamente un $25 \%$ de los casos. Aunque hay muchas posibles etiologías, a veces, no existe una asociación demostrable.

La presentación clínica más típica es dolor intenso y agudo, que a menudo se irradia desde el hombro hacia distal, por la cara lateral de brazo, pudiendo también irradiarse hacia la región cervical. Este dolor se exacerba con los movimientos de la extremidad y puede mantenerse por horas y hasta semanas (2 a 3). Generalmente después de disminuir el dolor, se observa cierto grado de paresia, la cual en la mayoría de los casos se extiende hasta el mes de haber comenzado los síntomas. En forma asociada se puede observar atrofia.

Los nervios más afectados son el axilar, el supraescapular, el toráxico largo y el músculocutaneo.

El diagnóstico diferencial de la neuritis braquial debe incluir causas de dolor agudo, asociado a paresia alrededor del hombro, siendo los mas comunes: lesión del manguito de los rotadores, síndrome de atrapamiento, capsulitis adhesiva y tendinitis calcica. Además se puede incluir las HNP cervicales, poliomielitis, herpes zoster, tumores de médula o plexo braquial y lesiones neurales compresivas de origen traumático.

Muchos de estos pueden ser excluidos usando sólo la clínica, sin embargo, en un porcentaje de pacientes se necesita exámenes de apoyo. Uno de estos ha sido la RM de hombro, la cual ha demostrado ser una excelente herramienta en el diagnóstico de la neuritis braquial, pudiendo además evaluar o descartar la mayoría de los diagnósticos diferenciales más frecuentes.

Helms y col(2) reportaron la utilidad de la RM, describieron una alta señal en secuencias T2 de los músculos supraespinoso, infraespinoso y deltoides. Los signos de atrofia eran evidentes dentro de pocas semanas de haber comenzado los síntomas. Uno de los falsos positivos más difíciles de descartar es una neuropatía compresiva del nervio supraescapular por un ganglion que se descomprime espontáneamente, dejando solamente visible el edema y la atrofia del músculo infraespinoso o supra e infraespinoso. Sin embargo, éste se asocia casi en un $100 \%$ a rotura del labrum posterior y además los síntomas comienzan en forma insidiosa.

La radiografía simple de hombro generalmente es normal, pudiendo demostrar una subluxación inferior de la cabeza humeral semanas después de haber comenzado los síntomas, debido al compromiso del músculo deltoides y del manguito de los rotadores ${ }^{(9)}$.

La electromiografía ha sido un examen de gran ayuda en la localización y confirmación del diagnóstico. Aunque los hallazgos son a menudo variables, típicamente revelarían una denervación aguda, lo que estaría indicando una neuropatía axonal|(10).

El tratamiento esta enfocado a disminuir los síntomas, para lo cual se utilizan analgésicos y en algunos pacientes corticoides. También la inmovilización es de gran ayuda en el manejo de los síntomas. Posteriormente se sugiere realizar una terapia de rehabilitación.

La neuritis braquial tiene un buen pronóstico a largo plazo, sin embargo, el tiempo que toma alcanzar una resolución completa es variable. Dentro de los factores de lentitud en la recuperación esta la intensidad y duración de los síntomas, numero de 
nervios afectados y el nivel de compromiso del plexo braquial.

\section{Bibliografía}

1. McCarty EC, Tsairis P, Warren RF. Brachial neuritis. Clin Orthop 1999; 368:37-43.

2. Helms CA, Martinez S Speer KP. Acute brachial neuritis (Parsonage-Turner syndrome): MR imaging appearance-report of three cases. Radiology 1998; 207:255-9.

3. Misamore GW, Lehman DE. Parsonage-Turner syndrome (acute brachial neuritis). J Bone Joint Surg Am 1996;78:1405-8.

4. Miller JD, Pruitt S, McDonald TJ. Acute brachial plexus neuritis: an uncommon cause of shoulder pain. Am Fam
Physician 2000; 62:2067-72.

5. Dillin L, Hoaglund FT, Scheck M. Brachial neuritis. J Bone Joint Surg Am 1985; 67:878-80.

6. Spillane JD. Localized neuritis of the shoulder girdle: A report of 46 cases in the MEF. Lancet 1943;2:532-535.

7. Parsonage MJ, Turner JWA. The shoulder-girdle syndrome. Lancet 1948; 1:973-978.

8. Beghi E, Kurland LT, Mulder DW, Nicolosi A: Brachial plexus neuropathy in the population of Rochester, Minnesota. Ann Neurol 1985; 18:320-323.

9. Bacevich BB. Paralytic brachial neuritis. Case report. J Bone Joint Surg 1976; 58:262-263.

10. Weikers NJ, Mattson $\mathrm{RH}$. Acute paralytic brachial neuritis. A clinical and electrodiagnostic study. Neurology 1969; 19:1153-1158. 